\title{
Dysphagia Management for People with Intellectual Disabilities: Practitioner Identified Processes, Barriers and Solutions
}

\author{
Dr. Darren D. Chadwick
}

The University of Wolverhampton

\begin{abstract}
Background: Dysphagia is a significant problem for adults with intellectual disabilities which has received sparse attention in the research literature. Little is currently documented about how dysphagia management operates and the barriers and associated strategies to address barriers utilised in practice.

Method: A brief survey containing open ended questions was completed by 38 practitioners about the way their service operates, the barriers they have faced in providing support around managing dysphagia and the solutions and strategies they have found useful.

Results \& Conclusions: The process of dysphagia management typically involved referral and assessment, development of an intervention strategy, communication and negotiation, education and training in safe dysphagia management and monitoring, evaluation and re-assessment. Barriers were numerous but stakeholder beliefs, knowledge and feelings underpinned many of them. Solutions varied but similarly were underpinned by good communication, building relationships, person centred practice and responsivity, pragmatism and innovation in training and disseminating dysphagia management information.
\end{abstract}

Keywords: Dysphagia, intellectual disabilities, practice, multidisciplinary working 


\section{Background}

Dysphagia, also referred to as eating, drinking and swallowing problems, can have serious repercussions for people with intellectual disabilities potentially leading to discomfort, poor nutritional status, dehydration, aspiration and choking (Aziz \& Cambell Taylor, 1999; Chadwick \& Jolliffe, 2009; Helfrich et al., 1986; Kennedy et al., 1997; Samuels \& Chadwick, 2006; Sheppard, 1995, 2006, 2010). Many of these can be life threatening or may lead to other life threatening problems and harm. Dysphagia management requires considerable effort from people with disabilities, paid and familial carers and social and health care services (Hollins et al., 1998; Chadwick \& Jolliffe, 2006). When harm occurs or is narrowly averted this can lead to distress for both the people with dysphagia and those who provide supports for them (Balandin et al., 2009; Ball et al., 2012; Chadwick et al., 2003).

With regard to people with dysphagia, individualised treatment programmes based on interdisciplinary team working have been acknowledged to be the most effective approach to intervention (NPSA, 2003, 2007). However no studies appear to have been conducted investigating the process of dysphagia management for adults with intellectual disabilities, or the nature of the involvement of different stakeholders. Practitioner support for dysphagia management is typically implemented by speech and language therapists (SLTs). Individualised interventions for adults with intellectual disabilities and dysphagia include altering positioning, modification of food and drink consistency, giving advice on equipment, and working on the physical environment through carer training (Chadwick et al., 2002; 2003). It is assumed that for interventions to be effective they must be adhered to. If interventions are implemented, and the individual with intellectual disabilities and his/her carers are trained in these person specific guidelines, it is assumed that the risk of dysphagia will be managed, and the potential for the negative health consequences to occur reduced. However, non-compliance with health advice, including dysphagia management programs has been identified as an issue (Chadwick et al., 2002, 2003; Crawford et al., 2007).

Chadwick et al. (2006) interviewed carers of people with intellectual disabilities and identified particular issues around modifying food and drinks to safe consistencies, achieving the agreed positioning during mealtimes, and in implementing support and prompting strategies. Aspects of dysphagia management relating to direct mealtime support that carers found more challenging, and which prompted more focused intervention strategies were identified. These included: pacing meals inappropriately and inconsistently; helping people to adequately relax and concentrate during meals; monitoring, observing and prompting people to pace themselves appropriately and take safe amounts of food and drinks during mealtimes. Additionally, carers mentioned time pressures, staff turnover, and insufficient re-examination of SLT management strategies by carers as hindering adherence. Ongoing support was also suggested for people with intellectual disabilities and dysphagia to help them to understand the reasoning behind management strategies.

Crawford et al. (2007) in a study of day service dysphagia support observed 27 people with intellectual disabilities at mealtimes. This study found that mealtime support was well followed by carers, possibly due to consistency amongst the staff team. Carers reported struggling more to support people who were more independent at mealtimes and also found that the importance placed by carers on particular strategies, 
leadership/guidance from a senior/experienced member of staff and the quality of training were influential in improving dysphagia support.

Non-adherence with dysphagia management strategies has been associated with adverse outcomes including chest infection, aspiration pneumonia, and death (Low et al., 2001). Despite the adverse effects, there have been few studies identifying the factors that may hinder or prevent adherence to dysphagia management strategies or the potential strategies that could act as solutions to management challenges. Nor have there been any empirically based descriptions of the process of dysphagia management with people with intellectual disabilities.

\section{Specific Aims}

The study aimed to identify:

1. The process of dysphagia management with adults with intellectual disabilities and those typically involved

2. The barriers to dysphagia management for adults with intellectual disabilities as identified by the health professionals working in this field

3. Current practices found to be successful in reducing these barriers

4. Strategies that have been less successful in reducing barriers.

\section{Method}

\section{Design}

This was a UK based exploratory descriptive study collecting qualitative information via a survey containing open ended questions.

\section{Participants \& Procedure}

A brief survey was distributed via an e-mailing list collated by the Royal College of Speech \& Language Therapists in collaboration with the NPSA ${ }^{1}$. The exact number of people who received the survey for completion is unknown as the survey was sent electronically, via the mailing list, accompanied by the request that the survey be passed on for completion to known experienced practitioners working with people with intellectual disabilities around dysphagia management. Background information was not gathered from the participants, other than their profession. This was done to enhance speed and likelihood of responding and reduce the time pressure on practitioner respondents. Hence, it was sent primarily to SLTs working with adults with intellectual disabilities and also to other health practitioners (e.g. dieticians, dysphagia trained nurses) who are involved in the management of dysphagia in people with intellectual disabilities. The survey contained open-ended questions asking respondents to report:

1. The way their dysphagia management process operated and who was involved in the last 10 dysphagia cases that the practitioner had been involved in;

2. The barriers and situations which have hindered management;

3. Methods utilised to resolve barriers so that dysphagia was more safely managed;

4. Strategies attempted to resolve barriers that were less successful. 
Respondents were not asked to list every barrier they had faced in dysphagia management in order to minimize the time taken to complete the survey and to encourage completion and return. Instead, participants were asked to report any specific hindrances that they had come across which they thought were typical of barriers to dysphagia management that practitioners working with adults with intellectual disabilities would confront. An opportunity sample of 38 practitioners working in dysphagia management (37 SLTs \& 1 Dietitian) participated in the study. The survey can be obtained from the first author on request. All participants worked within the UK National Health Service (NHS) context.

\section{Data Analysis}

Data was subject to thematic analytic approaches (Braun \& Clarke, 2006) involving familiarisation with the data, searching and coding of the survey text to identify themes within the data, and reviewing and confirming final themes and framework analysis (Pope et al. 2000) where a framework is devised from the initial themes to analyse subsequent data in a more structured and time efficient way. First the process of dysphagia management data was analysed and coded (See Figure 1). This resulted in a framework for the process of dysphagia management (Referral; Assessment; Implementation and training; Outcome Evaluation; Reviewing, monitoring and closing). Subsequently, the barriers identified and particular issues reported were organised according to this framework, assigned to the different stages of dysphagia management. However, some adaptations were required to accommodate barriers which did not easily fit into the framework. This resulted in the barriers being organised under the following organising structure: Pre-involvement; Assessment; Management Implementation; Ongoing Adherence; \& Organisational barriers.

Corresponding successful and unsuccessful strategies for overcoming barriers and unresolved issues were assigned to barriers at each of the framework stages.

Subsequently, the successful and unsuccessful strategies and the unresolved issues around the barriers where then thematically analysed. The stages of data analysis summarised incorporated: (1) Collation and transcription of the qualitative data; (2) Familiarisation with the data by repeated reading; (3) Identification of similarities (and differences) in the processes of dysphagia management detailed and development of process framework; (4) Collation of information about joint working (5) Classifying and coding the barriers data according to the dysphagia management process framework; (6) Adapting this framework to incorporate all the barriers and drawing links between barriers; (7) Description and coding of solutions; and (8) Description and coding of unresolved issues.

As checks of the trustworthiness of the qualitative analysis conducted, the findings regarding the barriers, solutions and process were member checked by a group of 14 senior dysphagia practitioners ( $>5$ years of working in the field) who agreed that both the summarised process commonalities outlined and barriers identified adequately represented their experiences. In addition, the thematic coding and process framework employed were subsequently checked and verified by an independent researcher experienced in qualitative analysis. 


\section{Findings}

\section{The process of dysphagia management}

Of the 38 practitioners who participated, $20(52.6 \%)$ provided details about how dysphagia management occurred in their community based service. Despite variations in the process of dysphagia management many similarities were found. The material gained from these responses was collated and can be seen in Figure 1.

Accompanying the process diagram is information detailing typical practice and issues facing practitioners at different stages in the process. These were derived from additional comments made by respondents and the barriers and solutions data where participants had included process information and issues.

As can be seen in Figure 1, the typical process of dysphagia management begins with the referral to either the multidisciplinary team (MDT), specialist dysphagia team or the SLT via an open or medical referral system. In either system GP approval for the referral was sought. Following this initial contact was made to determine the urgency of this referral which dictated typical response times. For urgent cases response times were reported as 48 hours, across all respondents who mentioned specific time frames. Response times ranged between 2-4 weeks across respondents if the case was considered less urgent.

The initial assessment was typically conducted by the SLT or by the SLT with other MDT members (e.g. Physiotherapist). Some services collected background information and assessed urgency simultaneously via a telephone screening questionnaire. Others posted a questionnaire to the primary carer to be completed prior to the initial home visit. Assessments were conducted over a number of visits initially involving questionnaires and interviews along with clinical observations. A range of other assessment techniques were used including cervical auscultation, pulse oximetry and videofluoroscopy. Usually assessments were made across a range of food and drink consistencies and in all places that the person spent time. A risk assessment component was sometimes incorporated into this and some services introduced interim guidelines following the first assessments.

The initial assessments were often discussed in MDT meetings. These assessments resulted in further referrals to other personnel and for further evaluations and medical investigations (e.g. dentist, radiologist, gastroenterologist, GP for medication changes). Clarification of team involvement also took place after the initial assessments, with the SLT most often mentioned as the lead practitioner with the physiotherapist and dietician also considered key, corroborating the findings reported regarding stakeholder levels of involvement in Table 1. Allocation as lead depended upon the results of the assessment and the nature of the particular problems the person had. Some disciplines were not always available (e.g. occupational therapy, dietetics). How to best support the person with intellectual disabilities and the results of additional investigations were discussed at MDT meetings where the progress of management was monitored and direction given. Three respondents mentioned having dysphagia care pathways in operation in their services. 
Figure 1: The typical process of dysphagia management and associated issues

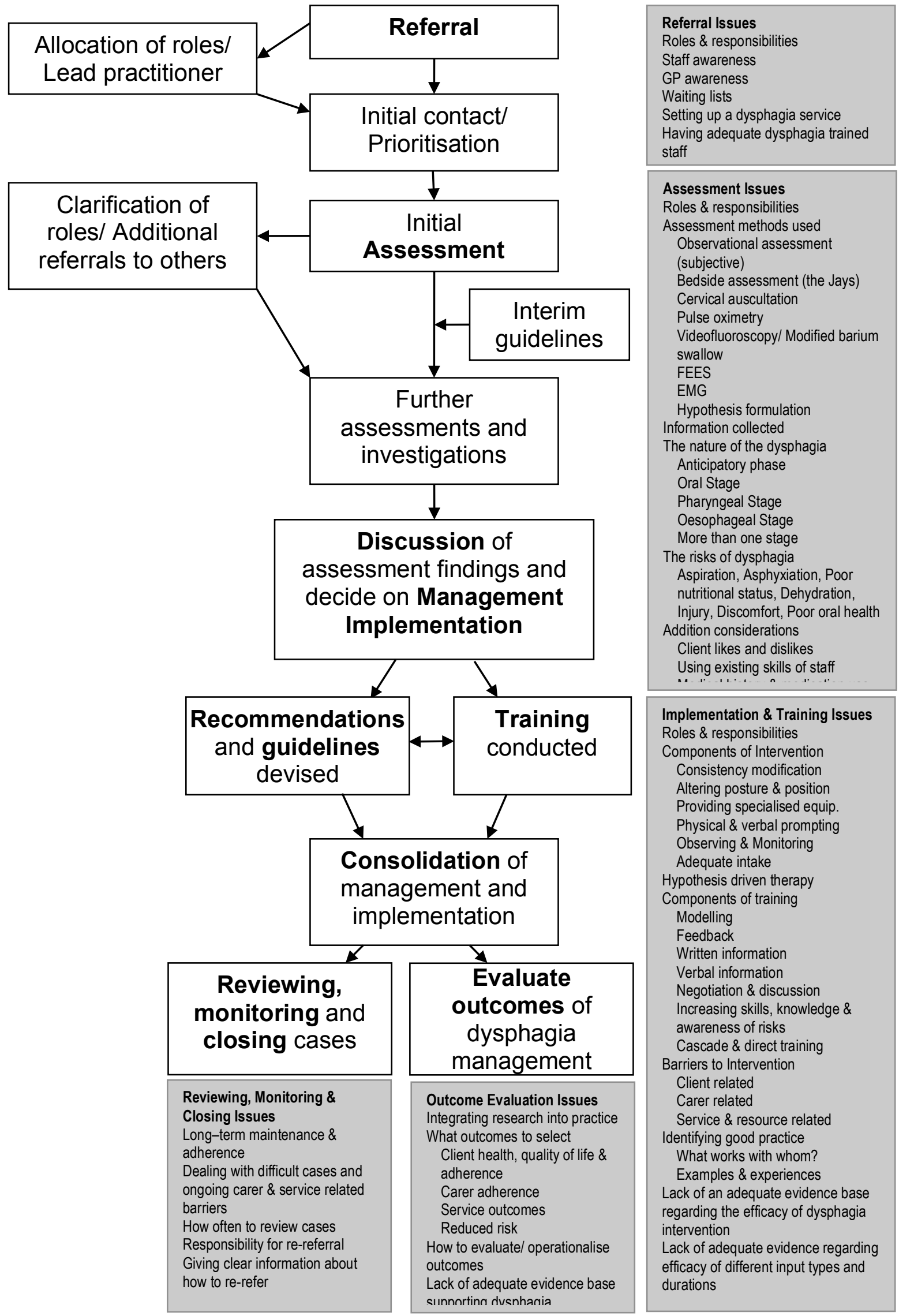


Once all assessments and investigations were complete and the involved practitioners had begun to formulate the recommendations, results were discussed with all stakeholders. At this point management implementation was agreed and specific strategies implemented. Specific and/or general training, often on a rolling program, was provided for paid or family carers. The specific training focused on the recommendations for the person they supported and all services provided this. Training progress was monitored and further training and guidance provided as required. Guidelines were written and copies were given to all stakeholders, these were sometimes incorporated into people's care plans. Accessible versions of guidelines were devised (e.g. simplified, laminated, and positioned in 'easy to get to' locations). The guidelines and ongoing safe practice were then consolidated.

Some respondents introduced charts for ongoing monitoring of chest infections, food preferences and problem foods, choking, fluid intake, coughing, gurgling, retching and vomiting. Risk assessments were indicated here to assess the stability of the situation following implementation. This risk information was used to inform the frequency of reviews at the end of management. Some services closed cases and gave clear guidance on how to re-refer, others reviewed at intervals based on the severity of the case and how well managed the dysphagia was (Larger intervals for less severe, more well managed cases).

Outcome evaluation was mentioned in a few instances though not often. These evaluations took the form of auditing knowledge and competence following training or interviewing staff to ascertain their perceptions of the quality of the advice given and the extent to which this was understood.

\section{Variation in dysphagia management practice}

In addition to the commonalities detailed above there were distinctions between services reported too. For example one service utilised dysphagia specialist SLTs who were not experts at working with people with intellectual disabilities who took the lead role if the person had an acquired dysphagia with the intellectual disability specialist taking the lead role if the dysphagia was developmental and considered primarily attributable to the neurological difference which also underpinned the person's intellectual disability. Other ways in which the services varied were: (i) whether the referral system was open or medical, only via a health professional; (ii) whether the SLT was always lead practitioner or whether it could be someone else within the multidisciplinary or specialist dysphagia team; (iii) the way the assessments were conducted; (iv) the way in which guidelines were written, presented and distributed; (v) whether cases were closed or open for review; and (vi) the extent to which MDT working occurred.

\section{Multidisciplinary \& Joint working}

The roles and responsibilities of the multidisciplinary health team members and other stakeholders in the dysphagia management process are shown in Table 1 together with the average frequency of involvement of the different stakeholders, based on respondents' previous 10 dysphagia cases. From the accounts of the study respondents, dysphagia management typically involved SLTs, dieticians and 
physiotherapists along with carers and the person with dysphagia him/herself. Many additional stakeholders were also identified as often being involved.

Table 1: Stakeholder ${ }^{1}$ involvement in dysphagia management

$\begin{array}{lrl}\text { Typically } & \text { Mean } \\ \text { Involved } & \text { frequency of } & \text { Roles \& Responsibilities of the } \\ \text { (\% Yes) } & \text { involvement } & \text { different stakeholders }\end{array}$

\begin{tabular}{|c|c|c|c|}
\hline \multirow{2}{*}{\multicolumn{4}{|c|}{ Health/Social Care }} \\
\hline & \multicolumn{2}{|c|}{ Professionals } & \\
\hline $\begin{array}{l}\text { Speech and Language } \\
\text { Therapist }\end{array}$ & 100.0 & $9.9(0.3)$ & $\begin{array}{l}\text { Effective assessment, intervention and } \\
\text { management of dysphagia within a } \\
\text { multidisciplinary context }\end{array}$ \\
\hline Dietitian & 78.9 & $4.8(2.6)$ & $\begin{array}{l}\text { Nutritional assessment, texture modification } \\
\text { advice, training on non-oral feeding, menu } \\
\text { planning, supplements }\end{array}$ \\
\hline Nurse & 63.2 & $3.9(2.0)$ & $\begin{array}{l}\text { Management and advice on epilepsy, } \\
\text { medication, effects of low hydration and } \\
\text { support in referring to appropriate professional }\end{array}$ \\
\hline Physiotherapist & 78.9 & $5.4(2.8)$ & $\begin{array}{l}\text { Assessment and advice on posture, stability, } \\
\text { comfort and function. Chest therapy to } \\
\text { minimise risk of chest infection }\end{array}$ \\
\hline Occupational Therapist & 63.2 & $3.0(2.2)$ & $\begin{array}{l}\text { Assessment of upper limb function, } \\
\text { adaptations to environment, assistive } \\
\text { equipment, seating and positioning }\end{array}$ \\
\hline Psychologist & 26.3 & $1.4(3.1)$ & $\begin{array}{l}\text { Assessment and advice on behavioural issues } \\
\text { relating to dysphagia }\end{array}$ \\
\hline $\begin{array}{l}\text { Care Manager/Social } \\
\text { Worker }\end{array}$ & 47.4 & $3.2(2.9)$ & $\begin{array}{l}\text { Co-ordination of care package to support the } \\
\text { person with dysphagia and care staff }\end{array}$ \\
\hline Radiologist & 31.6 & $3.1(4.0)$ & $\begin{array}{l}\text { Interpretation of videofluoroscopy, chest } \mathrm{X} \\
\text { rays and other radiological investigations }\end{array}$ \\
\hline General Practitioner & 73.7 & $7.0(2.7)$ & $\begin{array}{l}\text { Overview of health needs, assessment of } \\
\text { current health status, onward referral }\end{array}$ \\
\hline Gastroenterologist & 10.5 & $1.9(0.2)$ & $\begin{array}{l}\text { Clinical assessment, advice and intervention } \\
\text { on issues concerning severe dysphagia }\end{array}$ \\
\hline $\begin{array}{l}\text { Oral health promotion \& } \\
\text { Dentists }\end{array}$ & 10.5 & $5.5(1.8)$ & $\begin{array}{l}\text { Advice and intervention on oral health and } \\
\text { dysphagia related conditions }\end{array}$ \\
\hline \multicolumn{4}{|l|}{ Other Stakeholders } \\
\hline $\begin{array}{l}\text { Residential Staff \& } \\
\text { Managers }\end{array}$ & 100.0 & $8.0(1.8)$ & $\begin{array}{l}\text { Support individual, discuss and implement } \\
\text { dysphagia management guidelines and } \\
\text { identify changes to health status }\end{array}$ \\
\hline Day Centre Staff & 100.0 & $6.2(3.2)$ & As above \\
\hline Family Carer & 94.7 & $5.3(3.1)$ & As above \\
\hline $\begin{array}{l}\text { Client with intellectual } \\
\text { disabilities }\end{array}$ & 89.5 & $7.5(3.9)$ & $\begin{array}{l}\text { Report health changes and concerns to } \\
\text { relevant people if able, be involved in } \\
\text { developing and following dysphagia } \\
\text { management guidelines }\end{array}$ \\
\hline \multicolumn{4}{|l|}{ Others Mentioned } \\
\hline Pharmacist & 10.5 & $4.1(1.2)$ & Advice on and provision of medication \\
\hline Kitchen staff \& chefs & 10.5 & $5.5(6.4)$ & $\begin{array}{l}\text { Preparation of food in line with texture } \\
\text { modification guidelines }\end{array}$ \\
\hline
\end{tabular}

'Stakeholder' refers to all the people who are in any way involved in managing dysphagia. This includes the person with intellectual disabilities, all health professionals (in MDT, community and acute settings), carers (family members, adult placement providers, and day centre and residential support staff), and others (e.g. kitchen staff, pharmacist)

${ }^{2}$ Based on the respondents ten most recent dysphagia cases. 
One service was provided by dysphagia-trained nurses and these were always involved in dysphagia management. Family members, residential support staff and day centre staff were always involved when applicable. The person with intellectual disabilities was also often involved, although the extent of this involvement depended on the severity of their intellectual disability.

Respite services were not noted as involved in dysphagia management. Consultant psychiatrists, advocates, speech and language therapists from acute services and swallow clinic therapists were mentioned as having occasional involvement.

\section{Barriers to effective implementation}

In this second part of the results, the barriers faced at each stage of dysphagia management are identified, followed by the successful strategies implemented to overcome these barriers along with the unsuccessful strategies and finally the unresolved issues are presented. The barriers to dysphagia management can be seen in Box 1. All 38 practitioners participated in this part of the study. For those practitioners who did not provide information about the process of dysphagia management within their service or those involved in their last 10 cases, the barriers they reported were allocated to a stage of dysphagia management based on the details given in the accounts. If no details were given then no stage was allocated e.g. barriers directly relating to assessment versus organisational barriers, which may affect many stages of management

Box 1: Dysphagia Barriers Identified by Health Professionals (frequency; percentage)

\section{Pre-involvement Barriers}

Referral $(3 ; 7.9)$

Transition to adult services $(1 ; 2.6)$

SLT confidence/ expertise $(1 ; 2.6)$

\section{Assessment Barriers}

Conducting the assessment $(4 ; 10.5)$

Accessing videofluoroscopy $(8 ; 21.1)$

\section{Management Implementation Barriers}

Carer beliefs \& attitudes $(19 ; 50.0)$

Food \& drink modification $(17 ; 44.7)$

Carer knowledge \& understanding $(14 ; 36.8)$

Time issues $(3 ; 7.9)$

\author{
Ongoing Adherence Barriers \\ Staff adherence $(26 ; 68.4)$ \\ (Agency staff adherence $(3 ; 7.9)$ ) \\ Family adherence $(7 ; 18.4)$ \\ Client adherence $(5 ; 13.2)$

\section{Organisational Barriers} \\ Multidisciplinary working $(6 ; 15.8)$ \\ Acute Sector Barriers $(10 ; 26.3)$ \\ GP issues $(8 ; 21.1)$ \\ Resource issues $(5 ; 13.2)$
}

Note: Number in parentheses denote the number and percentage of practitioners mentioning these specific barriers

\section{Pre-involvement Barriers}

The barriers identified at this stage of dysphagia management included: lack of information at the point of transition from child to adult services; unclear, late or absent referral; and atrophy of dysphagia knowledge in trained SLTs who had few dysphagia cases. 
Problems were also reported in conducting the assessments with adults with intellectual disabilities. These included carers providing times and meals that were inappropriate for conducting the assessment, and the person with dysphagia being unable to co-operate with the assessment. Conducting videofluoroscopy (VF) was also reported as sometimes difficult with this client group. Reasons for this were difficulties accessing VF, compounded by the perceived reluctance of relevant personnel (GP, Acute sector SLT, Radiologist) to facilitate use of VF by adults with intellectual disabilities. Also mentioned were personal accompaniment to the VF (i.e. taken by unfamiliar carer) and the difficulty of conducting the VF with people with intellectual disabilities who cannot tolerate the procedure. Finally, the inappropriate nature of the VF equipment was also mentioned (e.g. the VF machine being inaccessible to wheelchair users).

\section{Management Implementation Barriers}

A number of reasons for failure to implement or follow management guidelines were identified. In particular 'knowledge and understanding' and 'attitudes and beliefs' were considered causative barriers faced at all stages of management. Often these were explicitly mentioned in relation to compliance issues.

Carer beliefs and attitudes were the most commonly mentioned barrier. Attitudinal and belief based barriers to the management of dysphagia permeated all parts of the process. These incorporate the beliefs held by paid carers, agency staff, families, the radiologist, GPs, acute sector SLTs and other acute sector personnel and the person with intellectual disabilities who had dysphagia. These attitudes and beliefs included:

- Disbelief, distrust, and lack of acceptance of both the diagnosis and the intervention strategy offered;

- Attributing dysphagia to an alternative cause (e.g. behaviour, choice, intellectual disability);

- Quality of life and wellbeing related beliefs (Belief that eating is an important part of life, question food and drink modification and exclusions);

- Over support due to fears of negative health consequences (e.g. choking);

- Under support due to beliefs about support (e.g. promoting independence);

- Negative attitude to SLT and others telling families how to care for their family members and poor response to constructive criticism.

Problems with describing and defining consistencies, a lack of accessible information about consistencies and inaccurate beliefs about how to modify food and drinks were all reported. Carers had difficulties modifying consistencies and using thickener appropriately. Some modified inconsistently and confused different consistencies. There was also opposition to modifying consistencies reported from staff, families, general practitioners and the person with intellectual disabilities themselves.

The knowledge and understanding of the management of dysphagia was reported as a problem underlying many of the other obstacles reported, especially pertaining to adherence. There was a lack of knowledge and understanding of potential risks, aspiration and asphyxiation, and the rationale for establishing guidelines. Procedural issues in dysphagia management were also reported as misunderstood by carers in 
some instances. Carers often did not know how to order equipment or who to call about different problems, and were reluctant to engage with training.

Carer communication was often reported as poor. This meant that necessary knowledge was not transferred between carers and that relevant health professionals were not called about problems and changes relevant to ongoing dysphagia management, for example a change in medication. Poor cascade training, where staff are expected to train others in managing dysphagia, was also reported.

Time issues also hindered management. Staff struggled to find time to provide 1-to-1 support for people with complex eating and drinking difficulties. Families had a lack of time to carry out recommendations. For the SLT, case load demands sometimes prevented response. It was clear from the data that time was an issue for those involved in promoting safe eating and drinking.

\section{Ongoing Adherence Barriers}

Staff, families and people with intellectual disabilities were reported as being unable, forgetting and/or refusing to follow dysphagia management recommendations. This was considered a fundamental barrier to safe eating and drinking by many respondents.

Staff adherence was most often reported as a problem by health practitioners. There were failures to implement and comply with written guidelines and, more specifically, with recommendations regarding texture modification, oral hygiene regimes and monitoring form completion. Inconsistent compliance was noted in individual staff members. Some chose when to follow the guidelines, while others changed written guidelines without consulting with the SLT. Inconsistent compliance was also cited within staff teams, where some staff followed the guidelines more effectively than others.

Respondents offered explanations for non-compliance in a number of instances. These primarily focused on two factors: (i) that staff lacked the skills and competence to implement the guidelines; and (ii) that their beliefs and fears inhibited their willingness to adhere. Carers, because they believed they knew the intellectually disabled person best, occasionally disbelieved SLTs, and were sometimes reported to be defensive when given advice regarding dysphagia management. Also carer apprehension and the fear that they may be supporting people incorrectly reportedly reduced adherence in some instances.

One of the more difficult aspects of dysphagia management was recognised to be supporting family carers to implement dysphagia guidelines, and unresolved barriers were most common among this group. Health professionals could not always use the same strategies with families to help ensure safe practice that they use with care staff.

Seven practitioners $(18.4 \%)$ raised concerns regarding family carers in the study. These comprised refusing assessment, refusing to carry out recommendations and time pressures outweighing the safety and personal dignity of the adults with intellectual disabilities who had dysphagia. These adherence issues were again attributed to beliefs, but in the case of the family carers this was more accurately 
characterised as disbelief. According to the practitioner reports ( $N=4 ; 10.5 \%)$, families sometimes disagreed with the recommendations, did not accept the associated risks of dysphagia and on occasion did not prioritise the safety and dignity of their adult children.

Finally the willingness of the adults with dysphagia to carry out the guidelines was reduced in situations where they missed foods that were considered unsafe, disliked thickener, and were reluctant to accept SLT advice. This resulted in them giving wrong information to inexperienced staff about what food and drinks were safe and unsafe. These barriers appear to be due to a dislike of appearing different to others and personal choice and preference regarding food and drinks.

\section{Organisational Barriers}

A number of organisational issues were apparent from the responses received. Lack of and ineffective MDT working were both reported. Absence of commitment, confidence, support and knowledge were all cited as explaining this lack of joint working, along with time limitations and poor communication.

Working within the acute sector was difficult due to medical staff having limited knowledge and understanding of dysphagia and of enteral feeding in people with intellectual disabilities. Situations were reported where people had been discharged prematurely. Medical personnel had claimed they had the expertise to assess the swallow, and community health personnel had not been consulted regarding decisions about eating, drinking and enteral feeding before discharge.

Working with the GP was occasionally reported as difficult due to their reluctance to be involved, refer and prescribe for dysphagia management. They were sometimes reported as disputing the necessity of SLT involvement and providing advice to families that contradicted SLT advice.

Finally, resource issues were a problem. These included insufficient staffing to cope with dysphagia referrals, lack of experience and expertise to intervene with very complex cases, difficulty acquiring appropriate utensils, and poor access to research literature and the lack of a research evidence base to support practice.

\section{Effective management strategies, ineffective strategies and unresolved issues}

\section{Effective strategies}

Table 2 details a summary of the main effective and ineffective strategies along with unresolved issues. In qualitatively analysing the strategies an overarching theme of 'effective communication' was generated. All of the strategies that were found effective in dysphagia management seemed to be underpinned by good communication and interaction by practitioners, or groups of practitioners, with those providing support around dysphagia or with the people with intellectual disabilities with dysphagia themselves. Effective liaison was considered essential throughout the management process. Specifically, this promoted working with people during transition and when in acute services. It was also noted as instrumental to overcoming problems around joint working and working with families and the GP. 
Table 2: Summarising practitioner identified effective, ineffective and unresolved strategies in dysphagia management

\begin{tabular}{|c|c|c|}
\hline $\begin{array}{l}\text { Effective Practice Strategies } \\
\text { (What works) } \\
\text { - Liaising effectively } \\
\text { - Providing innovative training } \\
\text { - Having open discussion } \\
\text { - Providing more accessible } \\
\text { documentation } \\
\text { - Involving others } \\
\text { - Being consistent } \\
\text { - Being pragmatic } \\
\text { - Using a person-centered } \\
\text { approach } \\
\text { - Long term approach } \\
\text { - Being tenacious }\end{array}$ & $\begin{array}{l}\text { Ineffective Strategies } \\
\text { (What NOT to do) } \\
\text { - Ignoring beliefs, values and } \\
\text { feelings of carers } \\
\text { - Giving information only once } \\
\text { - Relying solely on verbal and } \\
\text { written guidance } \\
\text { - Relying on written letters and } \\
\text { phone calls } \\
\text { - Relying on the self motivation of } \\
\text { - } \text { others } \\
\text { Giving constructive criticism when } \\
\text { the recipient appears defensive } \\
\text { - Arrange too many meetings } \\
\text { - Being patronizing, } \\
\text { confrontational, angry or nagging } \\
\text { Giving up }\end{array}$ & $\begin{array}{l}\text { Unresolved Difficulties } \\
\text { - Changing the beliefs \& } \\
\text { Improving adherence of: } \\
\text { (i) Staff } \\
\text { (ii) Families } \\
\text { (iii) People with } \\
\text { intellectual disabilities } \\
\text { - Long term maintenance } \\
\text { - Training agency staff } \\
\text { - Working effectively in the } \\
\text { acute sector } \\
\text { - Decision making around } \\
\text { enteral feeding } \\
\text { - SLT accessing: } \\
\text { (i) Research literature } \\
\text { (ii) Evidence base }\end{array}$ \\
\hline
\end{tabular}

Training was used to improve dysphagia management, to increase carer knowledge and understanding of dysphagia and to encourage adherence to safe management strategies. Training was used successfully to improve carers' ability to prepare food and drinks to a safe appropriate consistency and to address any negative attitudes to food and drink modification. In addition to providing dysphagia management advice, training was often aimed at addressing those attitudes and beliefs held by residential and day centre care staff that were thought to reduce the likelihood of uptake of safe eating and drinking practices. Successful strategies employed during training can be seen in Box 2.

Box 2: Successful strategies used during dysphagia management training

- Develop a relationship with carers

- Use pictures, models and videos to explain dysphagia

- Acknowledge carer beliefs and fears as legitimate

- Increase empathy for the person with dysphagia

- Frank discussion of risks and best interest

- Frank discussion of food and drink modification

- Spend time clearly describing and modelling how to achieve the correct safe consistencies

- Highlight the risk of non-compliance to staff

- Focus on the positive changes

- Video people and give feedback on their performance

- Involve people with intellectual disabilities in training their carers

- Train staff to explain when refusing to give unsafe items of food

- Problem solve and identify pragmatic solutions

- Require staff to sign guidelines to state that they have received training, they understand the guidelines and will implement them

- Train whole staff teams 
Some SLTs also mentioned the need for checks to be in place so that no untrained care staff member was permitted to support the person with dysphagia during mealtimes, e.g. requiring people to sign the individual guidelines once they had been trained in implementing them to indicate that they agree to follow them.

Training was seldom mentioned with reference to family carers $(\mathrm{N}=1,2.6 \%$ for family carers compared with $\mathrm{N}=11,28.9 \%$ for paid carers). Discussion and communication were seen as the main routes to supporting family carers to understand the necessity for proposed interventions. Training of acute sector staff to raise awareness of adults with intellectual disabilities and dysphagia in this population was said to be effective by one respondent. Involving other more experienced health and social care staff in the setting to provide leadership and a consistent message regarding the benefits of dysphagia intervention was successfully used to promote management.

Discussion was considered useful in all situations. In decision making and for ongoing management, discussion was a strategy used to address the beliefs, attitudes and understanding of carers and people with intellectual disabilities. It provided opportunities for stakeholders to voice their opinions and their fears, such dialogue was noted to facilitate the management process.

Being pragmatic when implementing management was identified as useful in assimilating guidelines and management into the person's life. For example, management was not easy and acknowledging that families may need a break and helping to create opportunities for respite was noted as effective by one SLT. Another pragmatic strategy used was suggesting time saving options like pre-thickened drinks.

SLTs often reported providing information in more accessible ways. For staff, families and people with intellectual disabilities more than one form of information may be required. Methods that had been successfully used to communicate the details and importance of dysphagia management included:

- Co-writing guidelines with clients;

- Using experiential learning to enable carers to better understand what it is like to have dysphagia and why changes are necessary;

- Using an interpreter with families where English was not their first language;

- Keeping guidelines in prominent and accessible locations (e.g. Simplified, laminated information on place mats, or on the kitchen wall);

- Using pictures, videos and models to help to get across information about the individual's dysphagia and associated risks, to aid management implementation and to supply feedback on performance;

- Providing sheets and leaflets detailing unsafe food and drinks and how to safely modify them; and

- Providing meal ideas, menus, and recipes for modified foods and drinks.

The provision of written information alone was noted as an insufficient method of presenting the guidelines. Ensuring that relevant information accompanied people in their everyday lives was found useful for maintaining safe practices across settings. For people with less severe intellectual disabilities this practice was reported to encourage individual ownership of dysphagia management. 
A person centred approach appeared to be considered crucial by respondents. Practitioners reported success when they took account of the needs, likes and capabilities of each individual. If the safe management of the dysphagia involved excluding particular foods and the individual was requesting them, SLTs and carers explained clearly the reason why the foods are unsafe. Using creative methods to make the texture of the modified diet as interesting as possible, incorporating safe versions of the person's desired foods were also reportedly important to success.

Finally, having a long-term approach to dysphagia management was important. Having the ability to give enough time to allow effective management, monitoring people for changes over time and being tenacious in trying to ensure safe management to reduce risk were all noted as vital to being an effective practitioner working in dysphagia management.

Ineffective strategies

It is important to acknowledge that the solutions presented here may not be effective in all situations and that a flexible, adaptive approach appears essential in dysphagia management. Communication and building relationships with others involved in the process appeared to underpin good dysphagia management. As well as being effective, training, discussion and liaison were all noted as ineffective by some respondents. Demonstration, repeating training, monitoring by asking questions rather than observing, providing prescriptive paper guidelines and using visual support materials in training, though found to be effective by some practitioners, were reported as unsuccessful by others.

Relying on written letters and phone calls to encourage carers and health personnel to be involved was not always found to be effective. Arranging frequent meetings was reported as counterproductive by some respondents, and was reported to hinder rather than support the management process. However, giving information only once and reliance on verbal and written guidance alone were both found to be ineffective in training people to manage dysphagia. It is important that sufficient information about the risks of non-implementation and the benefits of adherence are transmitted to motivate carers and people with intellectual disabilities to engage with safe practice or make informed decisions about what they eat and drink. Repetition of information, within reasonable limits was also advocated.

Ignoring the beliefs, values and feelings of carers was reported to undermine effective dysphagia management. Practitioners reported that it was important that management be a collaborative process, and that the feelings, attitudes and beliefs of carers be gauged and considered by the practitioner. This also included avoiding appearing overly critical of existing paid support or parental care when suggesting changes to help manage dysphagia. Practitioners reported times when they had been patronizing, confrontational, angry or nagging to people with dysphagia or their carers or had given up on providing input, due to lack of compliance. These were all identified as ways in which they themselves felt they had undermined and failed to integrate safe practice into the person's life. 
Unresolved Issues

The main unresolved issue reported was ensuring adherence with safer practice. The strategies implemented by health practitioners appeared, in the main, to focus on resolving this issue. This was successful in many instances, as detailed above but there were situations where staff, families and the people with intellectual disabilities themselves refused to follow guidelines to reduce risks.

Concerns were expressed about this issue as it resulted in their eating unsafe consistencies, refusing thickened drinks and misinforming new untrained staff about their dysphagia. Some chose not to adhere because they preferred unmodified food and drinks, did not like to appear different and hence were reluctant to accept management advice.

The strategies that proved successful in overcoming these barriers included: explaining basic anatomy, providing a more accessible version of guidelines for their own use and ensuring that each time carers refused to give the person an unsafe consistency they explained why. Involving people with intellectual disabilities in the training of their own support staff was sometimes effective. Finally in some instances when informed choice had been verified, using the processes detailed in the Mental Capacity Act (2005), the client's choice not to follow the guidelines was accepted.

In the case of individuals with severe dysphagia accompanied by severe or profound cognitive impairments, who were found to be unable to make their own decisions, the issues surrounding major life changes (for example moving to non-oral feeding) may need to be discussed by a group acting in the best interest of that person. The decision making process when considering moving someone to enteral feeding was found to be challenging for practitioners, with responsibilities reported as difficult to clarify.

In a small number of instances $(\mathrm{N}=2,5.3 \%)$ the disagreements between the health staff and family had not been resolved. It appeared that health professionals working in dysphagia management need clarification about the legally and morally correct course of action to take when family members continue to refuse to accept dysphagia management advice. Access to legal guidance and research information were concerns for some respondents $(\mathrm{N}=3,7.9 \%)$, and they perceived a lack of information being available to guide them.

Changes in the status of people, their dysphagia, their support and their environments could all make the long term maintenance of safe practice difficult. It was felt important that ongoing monitoring is in place, however, it was sometimes difficult to ensure that this was carried out. Accessing and training agency staff was a difficulty not always resolved by health practitioners. Finally, liaising with acute sector personnel did not always result in effective joint working between the community and acute sectors. 


\section{Discussion}

Though there were many commonalities in the process of dysphagia management reported by respondents, variations in multidisciplinary working were also apparent in the detailed accounts given. Further work is required to identify the range and types of multidisciplinary working that enable effective dysphagia management. It is very possible that variation in the types of management may still lead to effective practice however there remains a lack of empirical literature on the efficacy and effectiveness of dysphagia management for people with intellectual disabilities.

The identification of numerous stakeholders as involved in dysphagia management suggests that it is a highly complex task in terms of both the expertise required and the personnel and organisation involved. Lack of respite staff involvement was also reported and may be due to the relatively small number of respondents or because respite staff were included under the category of residential support staff.

Individual treatment programmes that include input from all relevant health and social care staff are widely acknowledged to be the most effective approach to intervention for people with dysphagia (Arvedson, 1993). Ideally, the team would include the care manager, GP, speech and language therapist, dietician, physiotherapist and occupational therapist with input from nursing, clinical psychology and dental services as necessary. The person with intellectual disabilities, family members, and care staff should play an active part in the process. From the accounts given in this study this approach appeared to be being followed.

This study has added to the existing literature about the barriers to implementing dysphagia management with adults with intellectual disabilities, as perceived by health practitioners involved in this type of work. In addition, this paper adds to the start that has been made towards identifying and sharing effective and ineffective practice in dysphagia management. This has indicated a number of strategies that may be of use to those currently working in dysphagia management and newly qualified dysphagia practitioners working with adults with intellectual disabilities.

The barriers identified were many and varied, they occurred at all points in the management and implementation process and corroborated barriers previously identified in the literature (e.g. Chadwick et al., 2006; Crawford et al., 2007) particularly around training and supporting carers and support and leadership from experienced staff in the setting facilitating dysphagia management. The beliefs, knowledge and feelings of clients, carers and other health professionals and multidisciplinary team members appeared to explain many of the difficulties health practitioners have in implementing dysphagia management for adults with intellectual disabilities (e.g. barriers to implementation of consistency modification and more general compliance). Dysphagia management is not a simple task but appears both time and effort intensive for those involved; this needs to be acknowledged by policy makers, healthcare providers and those directly involved in the management of dysphagia if effective and long lasting changes are to occur which will promote and maintain the safety of adults with intellectual disabilities and dysphagia.

Although modifying the consistency of food and drink is not an explicit category within the process of dysphagia management it is an important stage in the process of 
management that is implemented in many cases (Chadwick et al., 2003; Chadwick et al., 2014). This was mentioned frequently, in conjunction with attitudes and beliefs hindering the consistency modification strategies. It was therefore considered important enough to be addressed separately. The finding that many stakeholders, including those with intellectual disabilities themselves opposed modification of meals and drinks is a particularly troubling finding, given that the efficacy of consistency modification as a strategy to improve mealtime safety and physical wellbeing in people with intellectual disabilities who have dysphagia is as yet empirically unverified. If this intervention is unproven, why then is this being implemented with people who clearly do not wish for these changes to their meals? If consistency modification does indeed make eating and drinking safer and allow the bolus to be better controlled during deglutition then strong empirical evidence is needed to demonstrate this. This is challenging to do as SLTs often believe in consistency modification due to their clinical observations that it helps bolus management. Therefore, to ask for this intervention to be ceased or not implemented for a group of individuals identified with dysphagia who are randomised to a no-treatment group, as would be the case in a randomised control trial which would allow causative therapeutic benefits to be identified, raises ethical issues. These appear to be ethically challenging areas where more research is urgently needed.

Working towards concordance, rather than compliance by building relationships with stakeholders underpinned successful management according to participants, which accords with findings from prior research gathering information from direct care staff (Crawford et al., 2007; Chadwick et al., 2006). Findings suggest that all stakeholders need to be consulted, involved and listened to as part of management work. People with intellectual disabilities need to be fully involved in discussions about the reasoning behind the dietary changes and how they would like to be supported to achieve safer eating and drinking. Further studies are required to identify effective ways of working with people with intellectual disabilities who manage or contribute to the management of their own health. The social and stigma related issues around dysphagia interventions for people warrant much more consideration and exploration in future research with studies prizing the voices and views of those with ID and dysphagia advocated. Previous research has identified social impacts of having dysphagia, including stigma in other populations (e.g. Miller et al., 2006) and although some anecdotal accounts also exist for people with intellectual disabilities (e.g. Chadwick et al., 2006) this has yet to be fully explored for people with intellectual disabilities and their carers. Such stigma may lead engagement in unsafe eating and drinking practices in public or may lead to further social isolation or exclusion.

Families who may not have received help with eating and drinking problems during their family member's childhood can find it difficult to accept intervention when their child becomes an adult, as found previously in studies of carers (Chadwick et al., 2003, 2006). Results suggest that time should be spent listening to and acknowledging family members' feelings and beliefs if successful dysphagia management is to occur in the family home. Additional studies exploring ways of working with families towards safe, successful dysphagia management are needed.

Practitioner reports indicate that care staff require ongoing support from their managers, and from dysphagia practitioners to ensure that the guidelines are followed and that correct practice is maintained in the event of staff changes. This corroborates 
the findings from the study by Crawford et al. (2007) which reported that leadership from more experienced carers and good quality training by practitioners supported dysphagia management in day care settings. Carers also need adequate time and resources to enable them to support people effectively. Health professionals may need to work to acknowledge and address staff beliefs about dysphagia and the person with intellectual disabilities in order to facilitate effective management.

Effective strategies reported indicate that training in dysphagia management should include: raising awareness of dysphagia and its symptoms and the associated health risks; providing guidance on how to implement management strategies, paying particular attention to pacing and modifying food and drinks; addressing those beliefs and attitudes of carers which reduce their willingness to accept and implement dysphagia management advice. Recently there have been calls for additional consideration of risk and safety incident research involving paid carers (Hemsley et al., 2014).

The provision of written information alone was reported as insufficient. Information presented in more accessible ways devised in collaboration with and matched to the requirements of people who are intellectually disabled and their carers was reported as more effectively assimilated. Experiential learning, pictures, videos or models have been successfully used to enable carers to have a better awareness of dysphagia. Giving detailed information on safe and unsafe foods and food modification advice along with menus had proved useful in some cases. Time limitations appear to impact on management and it has been noted that successful management requires time to implement with additional time needed to consolidate training to ensure the information has been retained and to monitor health.

The results of this study indicate that it is essential that staff involved in direct management of dysphagia are made aware of their responsibility to ensure dysphagia guidelines are implemented. Findings suggest it would be good practice to put checks in place to ensure that untrained staff do not support people with dysphagia at mealtimes. Where safe management is not implemented the reasons why this occurred, the intervention provided and the ongoing risk to the individual should ideally be clearly documented. Investigations to ascertain whether there is a link between unmanaged and managed dysphagia and negative health consequences are needed.

The finding regarding uncertainty about the morally and legally correct course following non-adherence to safer eating and drinking practices raises the issue of safeguarding of people with intellectual disabilities with dysphagia (Office of Public Sector Information, 2006). This area needs further consideration within research and practice. If an individual's safety is witnessed to be at risk, due to carers disregarding advice or disagreeing about the guidance given around dysphagia management, and reasonable efforts to resolve the issue have proved unsuccessful, then a referral to safeguarding processes must be made by practitioners, carers or advocates (Hampshire Safeguarding Adults Board, 2012).

The situation may be more complex when a person with intellectual disabilities $\mathrm{him} /$ herself decides not to follow safe eating and drinking guidance, potentially placing themselves in danger (Dimon, 2006). Deciding on the operationalization of ongoing support around eating and drinking might be challenging to arrange under such 
circumstances, as health professionals cannot be compelled to act contrary to their professional judgement. Failure to act in the best interests of a person may lead to prosecution of healthcare professionals should an individual die who lacks capacity to make an informed decision around eating and drinking safely. Capacity to make an informed decision and best interest are likely to both need considerable consideration and assessment under such circumstances. People with intellectual disabilities who are found to lack capacity are likely to have a substitute decision-maker for legal, financial, health related issues. The role of these individuals in decision-making around dysphagia is not well understood, nor is the extent to which SLTs actually find themselves in the position as to having to assess capacity. Many of these areas have received scant attention within the research literature.

The finding that the move to non-oral feeding can be a complex and challenging process accords with recent research which has highlighted the challenges inherent in non-oral nutrition and decision-making around re-introduction of oral intake on the grounds of promoting individual wellbeing (Chadwick, 2014).

Effective communication appears to be the key to effective implementation which can take many forms. For many barriers no one single solution was identified. There appears no single correct way to resolve difficulties in dysphagia management; many of the strategies identified as effective by some practitioners were also identified as ineffective by other practitioners (e.g. training, discussion). Future work would be useful to unpack the differences in these situations, to identify whether they pertain to the person with intellectual disabilities, their carer, the health practitioner or organisational or environmental factors or any combination of these. It is possible that only by unpicking the specifics of the dysphagia management situation will the appropriateness of particular strategies become clear.

Training for staff in generic healthcare services may also be required. Challenges were faced regarding assessment of dysphagia, which involved health care staff lacking specific training in working with people with intellectual disabilities. It is possible that these barriers may be linked to implicit societal attitudes and perceptions that people with intellectual disabilities are not appropriate to receive equivalent healthcare to other groups, though barriers may also reflect lack of knowledge and experience (Emerson et al., 2011; Emerson \& Baines, 2010). More work is needed around the extent to which practitioners and those providing health and social care hold negative attitudes towards people with intellectual disabilities, the role this plays in the support people receive and how we can improve attitudes, experience and knowledge.

As noted in Table 1, the GP was reported to have a key role in dysphagia management in the community, with GPs being involved in over half of the dysphagia cases cited. Once a Day (Department of Health, 1999), a primary care handbook about people with an intellectual disability, states that a GP with a list of 2000 will have approximately 40 patients with an intellectual disability, of whom eight are likely to have severe intellectual disabilities. This is a very small percentage of their case load and as Aspray, Tyler and Quilliam (1999) reported, GPs may lack specialist knowledge and experience about the health needs of people with intellectual disabilities. The need for additional GP service time and resources to provide an effective service for people with complex health and communication problems remains a significant issue and has 
also been identified more recently (Slowie \& Martin, 2014). More work is needed regarding the successful involvement of generic healthcare services in the process of dysphagia management.

As degree of support needs appeared to influence whether or not the person with intellectual disabilities and dysphagia was involved in dysphagia management further work is needed to investigate the best ways of including people with higher support needs in dysphagia management. A significant gap that remains in the literature is the absence of the perspectives of people with intellectual disabilities of the management process, barriers and strategies used to improve mealtime safety. Prior research has clearly demonstrated that it is both possible and valuable to gather the views of people with intellectual disabilities about their own life experiences and to involve them in the research process (e.g. Garcia et al., 2014). For those whose expressive communication is more limited and whose severity of cognitive impairments preclude traditional methods of experience elicitation, observational research and gathering information from a carer as a proxy is indicated.

There are limitations to the study that should be considered when interpreting and drawing conclusions from the data. The response rate and sample size for the study were not extensive and so representativeness of the current findings cannot be guaranteed. It is unlikely that the data collected incorporates all current practice and all barriers to dysphagia management and further larger scale research is warranted conducted by research teams to overcome these issues. Furthermore, to encourage responding from often busy practitioners the only background information collected from participants was their profession. This raises the possible limitation that more than one practitioner from a particular service participated potentially inflating the apparent prevalence of practices that map onto those within that setting. Future studies could include more information about training and practice tenure to determine the role these factors have in practices and identification of barriers and solutions around dysphagia management. Gathering information about geographical location, composition of MDT team, workload and response rate would better enable representativeness, generalizability and transferability of the findings to be determined.

\section{Conclusion}

Dysphagia management is a complex process with many barriers to its effective execution and many strategies found to be effective in facilitating implementation. There is a clear need for further work to evaluate: (i) the efficacy and effectiveness of management strategies to promote safe eating, drinking and swallowing (i.e. compensatory strategies incorporating texture modification, altering positioning, using specialised utensils and pacing, prompting and monitoring appropriately); (ii) strategies to reduce and overcome barriers to implementing these strategies and to promote ongoing compliance (i.e. discussion, novel training approaches, liaising with relevant personnel); (iii) identification of client, family and staff characteristics that associate with better outcome when using particular interventions; and (iv) more work about negotiation and positive risk taking towards enhancing psychosocial as well as physical wellbeing for people with intellectual disabilities and dysphagia and their carers. Gathering together details of current practice, assessment, risk management, training packages and conducting case studies detailing difficult scenarios and methods used to overcome these difficulties would appear a fruitful way forward. 


\section{References}

Aspray, T., Francis, R.M., Tyler, S.P., Quilliam, S.J. (1999). Patients with learning disability in the community. BMJ, 318: 476-477.

Arvedson, J. (1993). Management of Swallowing Problems. In: J. C. Arvedson JC, Brosky L, editors. Pediatric Swallowing and Feeding: Assessment and Management. San Diago CA: Singular, 327-387.

Aziz, S. J. \& Cambell-Taylor, I. (1999). Neglect and abuse associated with undernutrition in long-term care in North America: Causes and solutions. Journal of Elder Abuse \& Neglect 10, 91-117.

Balandin, S., Hemsley, B., Hanley, L., \& Sheppard, J. J. (2009). Understanding mealtime changes for adults with cerebral palsy and the implications for support services. Journal of Intellectual \& Developmental Disability, 34, 197-206. doi:10. 1080/13668250903074489 Ball, S. L., Panter, S. G., Redley, M., Proctor, C.-A., Byrne, K., Clare, I. C. H., \& Holland, A. J. (2012). The extent and nature of need for mealtime support among adults with intel- lectual disabilities. Journal of Intellectual Disability Research, 56, 382-401. doi:10.1111/j.13652788.2011.01488.x

Braun, V. \& Clarke, V. (2006) Using thematic analysis in psychology. Qualitative Research in Psychology, 3, 77-101.

Chadwick, D.D. (2014). Balancing safety \& enjoyment: Recommending oral tastes for people with intellectual disabilities. Appetite, 81(1), 152-161.

Chadwick, D.D., Stubbs, J., Forvague, S., Anderson, D., Stacey, G. \& Tye, S. (2014) Training support staff to modify fluids to appropriate safe consistencies for adults with intellectual disabilities and dysphagia: An efficacy study. Journal of Intellectual Disability Research, 58(1), 84-98.

Chadwick, D. D. \& Jolliffe, J. (2009). A descriptive investigation of dysphagia in adults with intellectual disabilities. Journal of Intellectual Disability Research, 53(1), 29-43.

Chadwick, D. D., Jolliffe, J., \& Goldbart, J. (2002). Carer knowledge of dysphagia management strategies. International Journal of Language and Communication Disorders, 37(3), 345-358 Chadwick, D. D., Jolliffe, J., \& Goldbart, J. (2003). Adherence to eating and drinking guidelines for adults with intellectual disabilities and dysphagia. American Journal on Mental Retardation, 108(3), 202-211.

Chadwick, D. D., Jolliffe, J., Goldbart, J. \& Burton M. H. (2006). Barriers to caregiver compliance with eating and drinking recommendations for adults with intellectual disabilities and dysphagia Journal of Applied Research in Intellectual Disabilities, 19(2), 153-163.

Crawford, H., Leslie, P. \& Drinnan, M.J. (2007) Compliance with Dysphagia Recommendations by Carers of Adults with Intellectual Impairment. Dysphagia, 22(4), 326-334.

Department of Health. (1999) Once a day.

[http://webarchive.nationalarchives.gov.uk/+/www.dh.gov.uk/en/publicationsandstatistics/public ations/publicationspolicyandguidance/dh_4006868, Accessed 01/02/16]

Dimon, B. (2006). Mental capacity and professional advice in a patient with dysphagia. British Journal of Nursing, 15(10), 574-575.

Emerson E. \& Baines S. (2010) Health Inequalities \& People with Learning Disabilities in the UK. Learning Disabilities Observatory, Lancaster.

Emerson, E., Madden, R., Graham, H., Llewellyn, G., Hatton, C., Robertson, J. (2011). The health of disabled people and the social determinants of health. Public Health, 125, 145-147.

Garcia Iriarte, E., O'Brien, P., McConkey, R., Wolfe, M., \& O'Doherty, S. (2014). Identifying the key concerns of Irish persons with intellectual disability. Journal of Applied Research in Intellectual Disabilities, 27(6), 564-575.

Hampshire Safeguarding Adults Board (2012). Reducing the risk of choking for people with a learning disability: A Multi-agency review in Hampshire. 
Helfrich-Miller, K. R., Rector, and K. L., Straka, J. A. (1986). Dysphagia: It's treatment in the profoundly retarded patient with cerebral palsy. Archives of Physical and Medical Rehabilitation, 67, 520-525.

Hemsley, B., Balandin, S., Sheppard, J. J., Georgiou, A. \& Hill, S. (2014). A call for dysphagiarelated safety incident research in people with developmental disabilities. Journal of Intellectual \& Developmental Disability http://dx.doi.org/10.3109/13668250.2014.994172 Hollins, S., Attard, M. T., von Fraunhofer, N., McGuigan, S., \& Sedgwick, P. (1998). Mortality in people with learning disability: Risks, causes, and death certification findings in London. Developmental Medicine \& Child Neurology, 40, 50-56. doi:10. 1111/j.14698749.1998.tb15356.x

Kennedy, M., McCombie, L., Dawes, P., McConnell, K. N., \& Dunnigan, M. G. (1997). Nutritional support for patients with intellectual disabilities and nutrition/dysphagia disorders in community care. Journal of Intellectual Disability Research, 41(5), 430-436.

Low, J., Wyles, C., Wilinson, T., \& Sainsbury, R. (2001). the effect of compliance on clinical outcomes for patients with dysphagia on videofluoroscopy. Dysphagia, 16, 123-127.

The Mental Capacity Act 2005. [Access from http://www.legislation.gov.uk/ukpga/2005/9/contents]

Miller, N., Noble, E., Jones, D. \& Burn, D. (2006). Hard to swallow: dysphagia in Parkinson's disease. Age Ageing, 35(6), 614-8.

National Patient Safety Agency. (2003). Seven Steps to Patient Safety: the full reference guide. London: NPSA.

National Patient Safety Agency. (2007). Ensuring safer practice for adults with learning disabilities who have dysphagia. [http://www.nrls.npsa.nhs.uk/resources/?entryid45=59823 Accessed 01/02/16] Office of Public Sector Information. London. The Safeguarding Vulnerable Groups Act 2006 (England and Wales).

Pope C., Ziebland S. \& Mays N. (2000) Qualitative research in health care: Analysing qualitative data. British Medical Journal, 320, 114-116.

Samuels, R. \& Chadwick, D.D. (2006). Predictors of asphyxiation risk in adults with intellectual disability and dysphagia. Journal of Intellectual Disability Research, 50(5), 362-370.

Sheppard, J. J. (1995). Clinical evaluation and treatment. In S. R. Rosenthal, J. J. Sheppard, \& M. Lotze (Eds.), Dysphagia and the child with developmental disabilities: Medical, clinical, and family interventions (pp. 37-75). San Diego: Singular Publishing Group.

Sheppard, J. J. (2006). Developmental disability and swallowing disorders in adults. In J. Cichero \& B. Murdoch (Eds.), Dysphagia: Foundation, theory and practice (pp. 299-318).

Chichester, England: Wiley.

Sheppard, J. J. (2010). Foreign body ingestion (FBI). In H. N. Jones \& J. C. Rosenbek (Eds.), Dysphagia in rare conditions: An encyclopedia (pp. 219-226). Brisbane, Australia: Plural. Slowie, D. \& Martin, G. (2014). Narrowing the health inequality gap by annual health checks for patients with intellectual disability. British Journal of General Practice. DOI:

10.3399/bjgp14X677293

1. Note: Since this research was conducted the NPSA has ceased to exist, with the functions being passed to the UK NHS Commissioning Board. 